\title{
Typicality modulates attentional capture by object categories
}

\author{
Y. Isabella Lim ${ }^{1} \cdot$ Andrew Clement $^{1} \cdot$ Jay Pratt ${ }^{1}$ \\ Accepted: 19 December 2020 / Published online: 27 January 2021 \\ (C) The Psychonomic Society, Inc. 2021
}

\begin{abstract}
What we pay attention to in the visual environment is often driven by what we know about the world. For example, a number of studies have found that observers can adopt attentional sets for a particular semantic category. However, some objects are more typical members of a category than others. While previous evidence suggests that an object's typicality can influence the guidance of attention in visual search, it is unclear whether typicality can also influence the capture of attention. To test whether this is the case, participants were given a category of objects at the beginning of each trial. Then, a rapid serial visual presentation (RSVP) stream was presented at fixation, and participants had to indicate whether an object of the given category was present or absent from the stream. Importantly, a single flanker image also appeared above or below the central stream just before the target. This flanker could belong either to the same category as the target or a different category, and could be a typical or atypical exemplar of that category. Participants were less accurate at detecting the target when the flanker belonged to the same category as the target. Moreover, participants were even less accurate when the flanker was a typical exemplar of this category. Similar findings were observed when targets consisted of typical and atypical exemplars. Together, these findings indicate that the extent of attentional capture toward a distractor depends on whether the distractor matches the category and typicality of one's attentional set.
\end{abstract}

Keywords Attentional capture $\cdot$ Memory: Long-term memory $\cdot$ Categorization

What we pay attention to in the visual environment is dependent on a number of factors. For example, it is frequently debated whether attention is driven primarily by bottom-up factors, such as visual salience, or top-down factors, such as task goals. While bottom-up theories of attention suggest that visually salient objects will capture attention regardless of an observer's goals (Theeuwes, 1992; Yantis \& Jonides, 1984), top-down theories of attention suggest these objects will only capture attention when they share features with observers' attentional set (Folk et al., 1992; Folk \& Remington, 1998). This phenomenon, known as contingent capture, has been demonstrated in a variety of studies. For example, Folk et al. (2002) had participants search for a target letter that was defined by a particular color in a rapid serial visual presentation (RSVP) stream of images. On each trial, a uniquely colored distractor also appeared in the periphery. Critically, this

Y. Isabella Lim

isabella.lim@mail.utoronto.ca

1 Department of Psychology, University of Toronto, 100 St George St, Toronto, ON M5S 3G3, Canada distractor only captured attention when it shared the same color as the target. Such findings demonstrate that observers' attentional set plays an important role in determining which features capture attention.

Most studies of top-down attention have examined contingent capture for relatively simple features, such as color, abrupt onsets, and apparent motion (Folk et al., 1994). However, when we search for objects in our environment, we do not always have specific visual information about a target's features. In such cases, what we pay attention to in the visual environment is often driven by what we know about the world. Consistent with this notion, a number of studies have found that observers can adopt attentional sets for a particular semantic category (Baier \& Ansorge, 2019; Nako et al., 2014; Wu et al., 2013; Wyble et al., 2013; Yang \& Zelinsky, 2009). For example, combining eye-tracking with a visual search task, Yang and Zelinsky (2009) found that when participants adopt a categorical attentional set, targets belonging to that category are initially fixated at levels greater than chance. This allowed the authors to conclude that search guidance is biased toward items belonging to categorical attentional sets, even when specific visual information about a target's features is unknown. 
Although multiple studies have found that categorical attentional sets affect visual search, relatively few studies have assessed whether these attentional sets play a role in contingent capture. If observers can adopt attentional sets for a particular semantic category, distractors that belong to the same category as this attentional set should involuntarily capture attention. To test this, Wyble et al. (2013) presented an RSVP stream of object images at fixation in a variant of Folk et al.'s (2002) procedure. Each trial began with the name of a superordinate category. Participants then had to search for a target belonging to that category within the RSVP stream. Two flanker images also appeared prior to the target, one of which could belong to the same superordinate category as the target. Across three experiments, participants were worse at identifying the target when this critical flanker belonged to the same category as the target. Error rates were also higher in this condition, with participants incorrectly identifying the critical flanker as the target. These findings suggest that when observers adopt a categorical attentional set, attention is involuntarily shifted toward items that belong to the same category as this attentional set, even when those items are task irrelevant.

While Wyble et al.'s (2013) findings strongly suggest that contingent capture can occur at a categorical level, it is unclear whether the typicality of objects affects the extent of attentional capture. Many models of semantic memory suggest that semantic categories are organized around prototypes, or the most typical members of a category (e.g., Rosch, 1975). Typical exemplars are more likely to share features with other exemplars and are considered to be representative members of a category, although the process of determining typicality is often subjective (Hout et al., 2014). While typicality has been shown to affect processes such as object classification (Rosch et al., 1976), relatively few studies have assessed whether typicality plays a role in categorical attentional sets. In one study, Castelhano et al. (2008) manipulated the typicality of categorical targets in a visual search task. When participants adopted a categorical attentional set, they were faster to search for typical members of that category than atypical members. However, using eye-tracking, Castelhano et al. (2008) found that typicality influenced target verification time (i.e., the time to respond to a target after fixating on it), but not search guidance. Likewise, Maxfield et al. (2014) tested whether the typicality of categorical targets affects visual search. However, Maxfield et al. (2014) randomized their distractors to avoid high target-distractor visual similarity, which may have prevented typicality effects from emerging in Castelhano et al.'s (2008) study. Here, their experiment was successful in obtaining a typicality effect on search guidance, with participants fixating high-typicality targets earlier and more often than both medium-typicality and low-typicality targets. Thus, unlike Castelhano et al.'s (2008) findings, these findings suggest that an object's typicality can influence the guidance of attention in visual search.
In light of these conflicting results, it would be useful to provide further data on whether typicality plays an important role in categorical attentional sets. To do so, we tested whether the typicality of distractors play a role in the capture of attention. Doing so would demonstrate whether categorical attentional sets consist of typical members of a category, and if so, whether distractors that belong to the same category as observers' attentional set involuntarily capture attention. Like Wyble et al. (2013), we hypothesized that distractors that belong to the same category as observers' attentional set should be more effective at capturing attention, resulting in worse accuracy at detecting a target. Moreover, we hypothesized that typical members of this category should also be more effective at capturing attention, resulting in even worse accuracy. This should remain consistent regardless of whether targets consist of typical (Experiment 1) or atypical (Experiment 2) exemplars.

\section{Experiment 1}

The purpose of our first experiment was to test whether typical distractors that belong to the same category as observers' attentional set are more effective at capturing attention than atypical distractors. Participants searched for a target category of objects in an RSVP stream. On each trial, a single flanker image appeared above or below the central stream. This flanker could either belong to the same category as the target or a different category, and could be a typical or atypical exemplar of that category. When observers adopt a categorical attentional set, distractors that belong to the same category as this attentional set should capture attention. Moreover, if attention is biased toward typical exemplars, then typical distractors that belong to this category should capture attention more so than atypical distractors.

\section{Method}

\section{Participants}

To determine the appropriate sample size, we first conducted an a priori power analysis using $\mathrm{G}^{*}$ Power 3 (Faul et al., 2007). Assuming a small effect size $(f=0.1)$ and a moderate correlation between levels of our within-subjects factors $(\rho=0.5)$, this analysis indicated that a sample size of 36 participants would be necessary to observe a significant two-way interaction between flanker category and flanker typicality at $80 \%$ statistical power. Thirty-eight participants (28 females; mean age $=18.9$ years) were recruited from the University of Toronto and were compensated with $\$ 10$ or course credit. All participants reported normal or corrected-to-normal vision. Two participants were excluded because their overall accuracy was below $65 \%$. 


\section{Apparatus}

The experiment was programmed and run using PsychoPy2 (Peirce et al., 2019). Stimuli were presented on a 24-in. LCD monitor with a resolution of $2,560 \times$ 1,440 and a refresh rate of $144 \mathrm{~Hz}$. Participants sat $57 \mathrm{~cm}$ away from the monitor so that it subtended $53.3^{\circ}$ horizontally and $30^{\circ}$ vertically.

\section{Stimuli}

Stimuli consisted 480 images of objects. The images were presented in grayscale on a white background, and were equated in luminance using the SHINE toolbox (Willenbockel et al., 2010). Each image belonged to one of 24 object categories, and was either a typical or atypical exemplar of its category (see Fig. 1). Each category consisted of

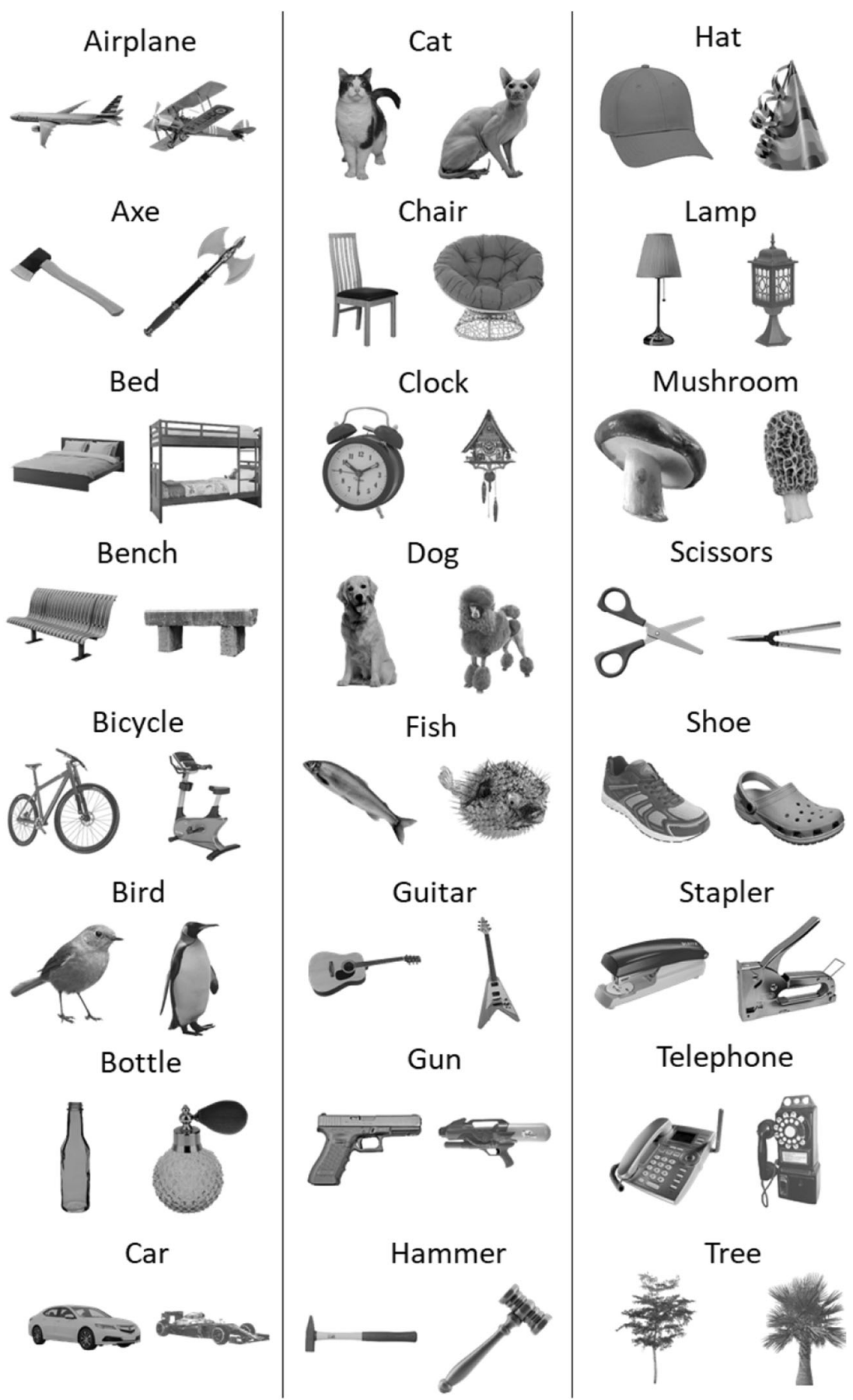

Fig. 1 Example images for each of the 24 object categories used in the present study. Typical exemplars are presented on the left side of each pair, and atypical exemplars are presented on the right 
10 typical and 10 atypical exemplars. Within the RSVP stream, each image subtended $2.3^{\circ} \times 2.3^{\circ}$. The flankers subtended $3^{\circ} \times 3^{\circ}$ and were presented $3.44^{\circ}$ above or below the central stream. Using Amazon Mechanical Turk, a total of 100 participants were recruited to rate one typical and one atypical exemplar from each category in terms of how typical the item was of its category. The rating scale ranged from 1 (highly atypical) to 7 (highly typical). A paired-samples $t$ test revealed that typical exemplars $(M=6.17, S D=0.79)$ were rated as significantly more typical than atypical exemplars $(M$ $=4.22, S D=1.11), t(99)=15.69, p<.001, \eta_{p}^{2}=.713$.

\section{Procedure and design}

Each trial was initiated by the participant with a key press. At the beginning of each trial, the name of one of the 24 object categories was randomly selected and presented in 40-point Lucida Console font for $600 \mathrm{~ms}$ (see Fig. 2). Each category had an equally likely chance of being selected. This category label identified the target category on a given trial. After the target category disappeared, an RSVP stream of nine images was presented at fixation. Each image was presented for $100 \mathrm{~ms}$. One of the images was the target (a typical object randomly selected from the displayed category), which was present on half of trials and absent on the other half (randomly determined). The target could randomly appear at positions 5, 6, 7, or 8 in the RSVP stream. Two images prior to the target, a single flanker image appeared above or below the RSVP stream. Within each testing session, it was equally likely that the flanker would appear above or below the RSVP stream, and would belong to the same category or a different category than the target (randomly determined). Importantly, the flanker was also equally likely to be a typical or atypical member of its category (also randomly determined). On target-absent trials, the flanker would still appear as noted above. All nontarget images in the stream were randomized and could not belong to the target category.

At the end of the stream, participants were required to make a key-press response indicating whether the target was present or absent within the stream ("z" or " $m$ " key). Key-press response mappings were counterbalanced between participants. Participants were instructed to only respond based on what they saw in the central stream. No feedback was provided. Each participant completed 15 practice trials, followed by 320 experimental trials in a single block.

\section{Results}

The mean percentage of correct responses on target-present trials was analyzed with a 2 (flanker category: same, different) $\times 2$ (flanker typicality: typical, atypical) within-subjects analysis of variance (ANOVA; see Fig. 3a). Replicating the findings of Wyble et al. (2013), we observed a significant main effect of flanker category, $F(1,35)=19.3, p<.001, \eta_{p}^{2}=.355$. Specifically, same-category flankers $(M=66 \%, S D=22 \%)$ resulted in worse accuracy than different-category flankers $(M=$ $81 \%, S D=8 \%)$. Thus, participants were more likely to make false negatives when the flanker belonged to the same category as the target. There was no significant main effect of typicality, $F(1,35)=4.06, p=.052, \eta_{p}^{2}=.104$. However, there was a significant interaction between flanker category and typicality,

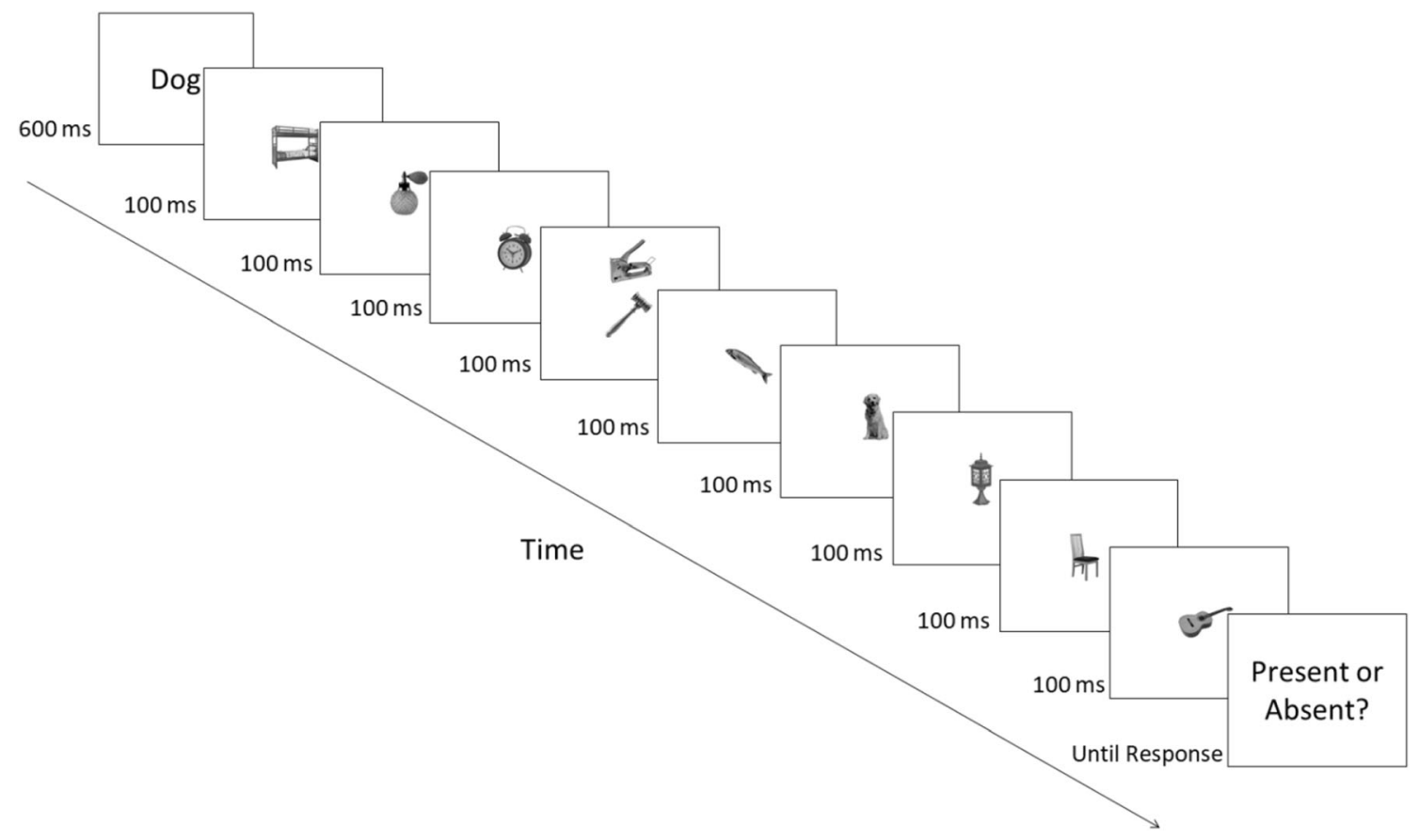

Fig. 2 Sample trial sequence in Experiment 1 

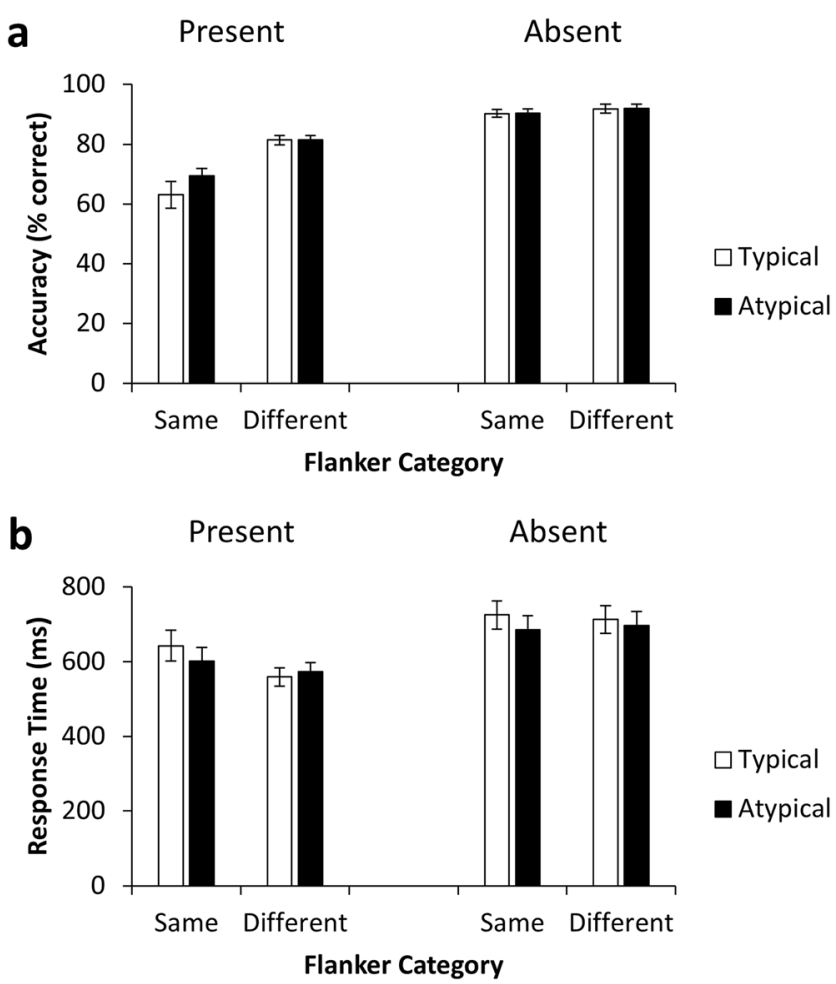

Fig. 3 a Percentage of correct responses on target-present (left) and target-absent (right) trials (see Appendix 1 for a detailed figure). Error bars represent the standard error of the mean for each condition. b Response time on target-present (left) and target-absent (right) trials. Error bars represent the standard error of the mean for each condition

$F(1,35)=4.68, p=.037, \eta_{p}^{2}=.118$. Paired-samples $t$ tests revealed a significant difference between same-category typical $(M=63 \%, S D=27 \%)$ and same-category atypical flankers $(M=$ $69 \%, S D=14 \%), t(35)=2.31, p=.027$, while no difference was found for different-category typical $(M=81 \%, S D=9 \%)$ and different-category atypical flankers $(M=81 \%, S D=8 \%), t(35)=$ $0.05, p=.959$. These findings suggest that distractors that belonged to the same category as observers' attentional set captured attention, resulting in worse accuracy at detecting the target. Of these distractors, typical exemplars captured attention more so than atypical exemplars, resulting in even worse accuracy. The mean percentage of correct responses on target-absent trials was also analyzed with a 2 (flanker category: same, different) $\times 2$ (flanker typicality: typical, atypical) within-subjects ANOVA (see Fig. 3a). Accuracy on these trials showed a significant main effect of flanker category, $F(1,35)=5.24, p=.028, \eta_{p}^{2}=.130$, with same-category flankers $(M=90 \%, S D=9 \%)$ resulting in worse accuracy than different-category flankers $(M=92 \%, S D=$ $9 \%$ ). Thus, participants were also more likely to make false positives when the flanker belonged to the same category as the target. There was neither a significant main effect of typicality, $F(1,35)=0.03, p=.865, \eta_{p}^{2}=.001$, nor a significant interaction between flanker category and typicality, $F(1,35)=0.01, p=$ $.963, \eta_{p}^{2}<.001$.
Although our main measure of interest was accuracy, we also analyzed response times. Response times faster than $200 \mathrm{~ms}$ were removed from analysis. The mean response time on target-present trials was analyzed with a 2 (flanker category: same, different) $\times 2$ (flanker typicality: typical, atypical) within-subjects ANOVA (see Fig. 3b). We observed a significant main effect of flanker category, $F(1,35)=7.18, p=.011, \eta_{p}^{2}=.170$. Specifically, samecategory flankers ( $M=623 \mathrm{~ms}, S D=232 \mathrm{~ms})$ resulted in slower response times than different-category flankers $(M=566 \mathrm{~ms}, S D$ $=148 \mathrm{~ms}$ ). This provides converging evidence for the present findings, suggesting that same-category distractors captured attention more so than different-category distractors. Importantly, this also suggests that the present findings were not due to a speedaccuracy trade-off. In addition, there was neither a significant main effect of typicality, $F(1,35)=.83, p=0.37, \eta_{p}^{2}=.023$, nor a significant interaction between category and typicality, $F(1,35)=3.25, p=.08, \eta_{p}^{2}=.085$. Response times on targetabsent trials showed a significant main effect of typicality, $F(1$, $35)=4.74, p=.036, \eta_{p}^{2}=.119$, with typical flankers $(M=719 \mathrm{~ms}$, $S D=226 \mathrm{~ms}$ ) causing slower responses than atypical flankers ( $M$ $=692 \mathrm{~ms}, S D=211 \mathrm{~ms}$ ). There was neither a significant main effect of flanker category, $F(1,35)=0.01, p=.963, \eta_{p}^{2}<.001$, nor a significant interaction between flanker category and typicality, $F(1,35)=1.27, p=.268, \eta_{p}^{2}=.035$.

\section{Item analysis}

Further exploratory analyses were conducted to examine whether object typicality ratings influence the extent of attentional capture. Specifically, we wanted to test whether objects rated as more typical captured attention more so than objects rated as less typical. To test this, we performed a Spearman's correlation test between two variables: (1) an object's mean typicality rating and (2) accuracy on trials where an object was used as a same-category flanker. Across all object categories, we observed a weak negative correlation between typicality ratings and accuracy, $\rho=-.115, p=.017$. This suggests that objects rated more highly (i.e., more typical) result in lower accuracy, providing converging evidence that typical distractors capture attention more so than atypical distractors.

\section{Discussion}

In Experiment 1, typicality influenced attentional capture by object categories. Participants were slower and less accurate at detecting the target when the flanker belonged to the same category as the target. Thus, distractors that belonged to the same category as observers' attentional set captured attention (Wyble et al., 2013). Moreover, participants were even less accurate when the flanker was a typical exemplar of this category. Thus, typical distractors captured attention more so than 
atypical distractors. The extent of attentional capture correlated with object typicality ratings, providing converging evidence for these effects. Together, these findings suggest that typicality plays an important role in categorical attentional sets.

\section{Experiment 2}

Experiment 1 showed that when observers adopt a categorical attentional set, distractors that belong to the same category as this attentional set capture attention. Moreover, typical distractors that belong to this category capture attention more so than atypical distractors. However, because targets always consisted of typical exemplars, it is possible that these results are due to the visual similarity between targets and distractors, with typical exemplars capturing attention because they share features with the target. Thus, the purpose of Experiment 2 was to address this potential confound. Participants completed the same task as in Experiment 1. However, targets always consisted of atypical exemplars. If attention is biased toward typical exemplars, then typical distractors that belong to the same category as observers' attentional set should capture attention more so than atypical distractors, even when targets are atypical.

\section{Method}

\section{Participants}

Eighty-one participants ( 34 females; mean age $=35.6$ years) were recruited from Amazon Mechanical Turk and were compensated with $\$ 4.50$. All participants reported normal or corrected-to-normal vision. Forty-five participants were excluded because their overall accuracy was below $65 \%$.

\section{Apparatus}

The experiment was programmed and run using PsychoPy2 (Peirce et al., 2019). Stimuli were displayed on a web browser window. Web browser, computer, and screen dimensions were varied across participants.

\section{Stimuli}

The stimuli used in Experiment 2 were identical to those of Experiment 1.

\section{Procedure and design}

The procedure and design were identical to those of Experiment 1. However, all targets in Experiment 2 were atypical exemplars. Trials were also separated into five blocks of 64 trials each, with short breaks between each block.

\section{Results}

The mean percentage of correct responses on target-present trials was analyzed with a 2 (flanker category: same, different) $\times 2$ (flanker typicality: typical, atypical) within-subjects ANOVA (see Fig. 4a). We observed a significant main effect of flanker category, $F(1,35)=15.35, p<.001, \eta_{p}^{2}=.305$, with same-category flankers $(M=58.6 \%, S D=15.7 \%)$ resulting in worse accuracy than different-category flankers $(M=67 \%, S D$ $=12.3 \%$ ). This provides further evidence in support of Wyble et al.'s (2013) findings that same-category distractors capture attention more so than different-category distractors. There was also a significant main effect of flanker typicality, $F(1,35)=$ $5.20, p=.029, \eta_{p}^{2}=.129$, with typical flankers resulting in worse accuracy $(M=61.6 \%, S D=16.4 \%)$ than atypical flankers $(M=63.9 \%, S D=12.7 \%)$. More importantly, we observed a significant two-way interaction between flanker category and flanker typicality, $F(1,35)=20.11, p<.001, \eta_{p}^{2}=$ .365. Paired-samples $t$ tests revealed a significant difference between same-category typical $(M=55.1 \%, S D=16.9 \%)$ and same-category atypical flankers $(M=62.1 \%, S D=$ $13.8 \%), t(35)=4.65, p<.001$. However, there was no significant difference between different-category typical $(M=68.2 \%$, $S D=13.2 \%)$ and different-category atypical flankers $(M=$ $65.8 \%, S D=11.4 \%), t(35)=1.74, p=.091$. Importantly, this
a
Present
Absent

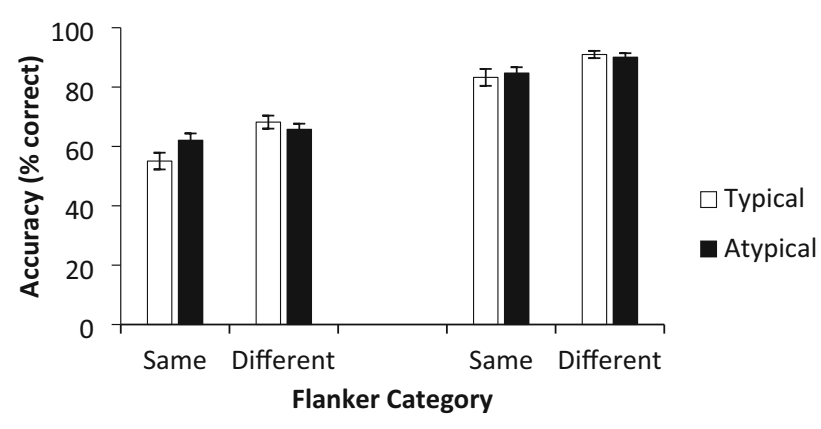

b

Present

Absent

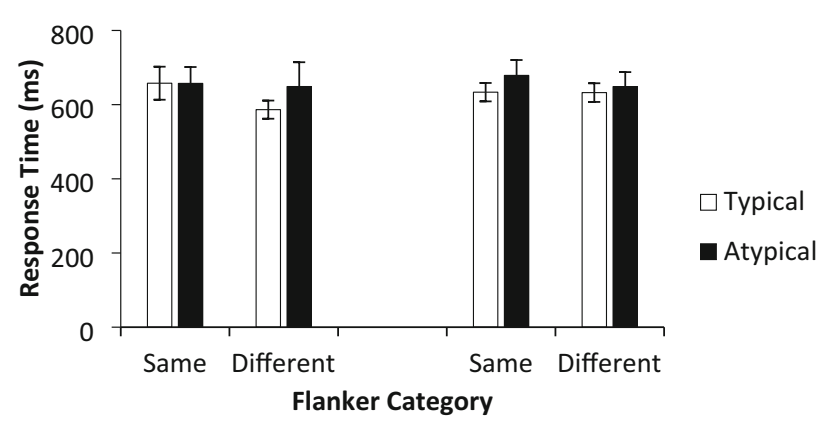

Fig. 4 a Percentage of correct responses on target-present (left) and target-absent (right) trials (see Appendix 2 for a detailed figure). Error bars represent the standard error of the mean for each condition. b Response time on target-present (left) and target-absent (right) trials. Error bars represent the standard error of the mean for each condition 
suggests that typical distractors that belonged to the same category as observers' attentional set captured attention more so than atypical distractors that belonged to this category. The mean percentage of correct responses on target-absent trials was also analyzed with a 2 (flanker category: same, different) $\times 2$ (flanker typicality: typical, atypical) within-subjects ANOVA (see Fig. 4a). We observed a significant main effect of flanker category, $F(1,35)=11.48, p=.002, \eta_{p}^{2}=.247$, with same-category flankers $(M=84 \%, S D=14.6 \%)$ resulting in worse accuracy than different-category flankers $(M=90.5 \%$, $S D=7.7 \%$ ). There was neither a significant main effect of flanker typicality, $F(1,35)=.10, p=.754, \eta_{p}^{2}=.003$, nor a significant interaction between flanker category and flanker typicality, $F(1,35)=1.72, p=.198, \eta_{p}^{2}=.047$. Response times did not differ as a function of flanker category or flanker typicality on target-present or target-absent trials, $p \mathrm{~s} \geq .130$.

\section{Item analysis}

Further exploratory analyses were conducted to examine whether object typicality ratings influence the extent of attentional capture. To test this, we performed a Spearman's correlation test between two variables: (1) an object's mean typicality rating and (2) accuracy on trials where an object was used as a same-category flanker. Across all object categories, we did not observe a correlation between typicality ratings and accuracy, $\rho=-.05, p=.560$.

\section{Cross-experiment analysis}

To determine whether accuracy for target-present trials differed between Experiment 1 and 2, we conducted a 2 (experiment: Experiments 1,2) $\times 2$ (flanker category: same, different) $\times 2$ (flanker typicality: typical, atypical) mixed-model ANOVA. Experiment was entered as a between-subjects factor, and target presence, flanker category, and flanker typicality were entered as within-subjects factors. We observed a significant main effect of experiment, $F(1,70)=17.46, p<.001, \eta_{p}^{2}=.20$, with worse overall accuracy in Experiment $2(M=62.8 \%, S D=$ $14.7 \%)$ compared with Experiment $1(M=73.8 \%, S D=$ $18 \%)$. This finding confirms that searching for atypical targets is more difficult than searching for typical targets. We also observed a significant main effect of flanker category, $F(1$, $70)=33.66, p<.001, \eta_{p}^{2}=.325$, with worse accuracy when flankers were of the same category $(M=62.8 \%, S D=19.2 \%)$ compared with the different category $(M=73.8 \%, S D=$ $12.7 \%)$. The main effect of flanker typicality was also significant, $F(1,70)=8.49, p=.005, \eta_{p}^{2}=.108$, with typical flankers $(M=66.9 \%, S D=20 \%)$ resulting in worse accuracy than atypical flankers $(M=69.7 \%, S D=14 \%)$. There was also a significant interaction between flanker category and flanker typicality, $F(1,70)=19.05, p<.001, \eta_{p}^{2}=.214$. Among same-category flankers, typical flankers $(M=59.1 \%, S D=$ $22.6 \%)$ resulted in worse accuracy than atypical flankers $(M=$ $65.8 \%, S D=14.5 \%), t(71)=4.26, p<.001$. This was not the case among different-category flankers, $t(71)=1.22, p=.228$. All other interactions were insignificant, $p \mathrm{~s} \geq .104$.

Accuracy for target-absent trials was also tested in a 2 (experiment: Experiments 1,2) $\times 2$ (flanker category: same, different) $\times$ 2 (flanker typicality: typical, atypical) mixed-model ANOVA. We did not observe a significant main effect of experiment, $F(1,70)=3.45, p=.068, \eta_{p}^{2}=.047$. However, we observed a significant main effect of flanker category, $F(1,70)=15.78, p<$ $.001, \eta_{p}^{2}=.184$, with worse accuracy when flankers were of the same category $(M=87.1 \%, S D=12.3 \%)$ compared with the different category $(M=91.2 \%, S D=8.2 \%)$. There was also a significant interaction between experiment and flanker category, $F(1,70)=5.68, p=.02, \eta_{p}^{2}=.075$. For Experiment 1, samecategory flankers $(M=90.2 \%, S D=8.5 \%)$ resulted in worse accuracy than different-category flankers $(M=91.9 \%, S D=$ $8.6 \%), t(71)=2.25, p=.028$. This was also the case for Experiment 2, with worse accuracy for same-category flankers $(M=84 \%, S D=14.6 \%)$ compared with different-category flankers $(M=90.5 \%, S D=7.7 \%), t(71)=4.35, p<.001$. No other main effects or interactions were significant, $p \mathrm{~s} \geq .304$.

\section{Discussion}

In Experiment 2, typicality again influenced attentional capture by object categories. As in the previous experiment, participants were less accurate at detecting the target when the flanker belonged to the same category as the target. Thus, distractors that belonged to the same category as observers' attentional set captured attention (Wyble et al., 2013). Moreover, participants were even less accurate when the flanker was a typical exemplar of this category. Thus, typical distractors captured attention more so than atypical distractors, even when targets were atypical. Participants displayed worse overall accuracy than in Experiment 1, suggesting that searching for atypical targets is more difficult than searching for typical targets (Maxfield et al., 2014). Again, these findings suggest that typicality plays an important role in categorical attentional sets.

\section{General discussion}

A number of studies have found that observers can adopt attentional sets for a particular semantic category (Baier \& Ansorge, 2019; Nako et al., 2014; Wu et al., 2013; Wyble et al., 2013; Yang \& Zelinsky, 2009). However, some objects are more typical members of a category than others. While previous evidence suggests that an object's typicality can influence the guidance of attention in visual search (Maxfield 
et al., 2014), it is unclear whether typicality can also influence the capture of attention. To test whether this is the case, we had participants search for a target category of objects in an RSVP stream. On each trial, a single flanker image appeared above or below the central stream. This flanker could either belong to the same category as the target or a different category, and could be a typical or atypical exemplar of that category. Consistent with Wyble et al.'s (2013) findings, participants were less accurate at detecting the target when the flanker belonged to the same category as the target. Moreover, participants were even less accurate when the flanker was a typical exemplar of this category. These findings demonstrate that when observers adopt a categorical attentional set, typical distractors that belong to the same category as this attentional set capture attention more so than atypical distractors that belong to this category. Furthermore, in Experiment 2, we show that these results are not purely due to the visual similarity between targets and distractors.

Importantly, the present findings provide support for the interaction between spreading activation and the allocation of attention. Spreading activation is the notion that when a concept is processed in semantic memory, activation spreads along a network of related nodes (i.e., concepts), with closely related nodes receiving the greatest activation (e.g., Collins \& Loftus, 1975). The further two nodes are apart, the less activation is received by the second node. Previous research suggests that when observers adopt a categorical attentional set, this spread of activation can influence where attention is directed (Moores et al., 2003; see also Maxfield \& Zelinsky, 2012; Schmidt \& Zelinsky, 2009). Consistent with spreading activation models, our results suggest that same-category distractors capture attention more so than different-category distractors. Spreading activation models would posit that this is because when one adopts a categorical attentional set, members of this category are more strongly activated relative to members of different categories. Importantly, this also has implications for an object's typicality. Typical exemplars are thought to be more closely related to their category within the semantic network, resulting in greater activation relative to atypical exemplars. This should then allow for greater attentional capture, which is what we observed in the present study. Our findings from Experiment 2 also suggest that despite searching for atypical exemplars, typical distractors belonging to the categorical attentional set still capture attention more so than atypical distractors do.

The present findings are also consistent with many theoretical models of attention (e.g., Duncan \& Humphreys, 1989; Wolfe, 1994), which suggest that top-down factors, including attentional sets, can influence which features in the visual world are attended to. According to such models, an activation map of the visual world is constructed based on the strength of top-down and bottom-up factors, which then determines where attention is deployed. Attention is prioritized toward objects based on how closely they match one's attentional set, either in terms of features or exemplars. In line with these models, typical exemplars of categories are prioritized and more able to capture attention, perhaps because typical exemplars contain features that most closely match their category's attentional template. Even when searching for atypical targets, typical distractors of the searched category still captured attention most, suggesting that categorical templates are biased toward what is typical of a category. Similar research on template matching may provide a good explanation for the mechanisms responsible for these typicality affects.

By adopting a categorical attentional set, a representation of the target is thought to be maintained in visual working memory. This representation is suggested to activate neurons that code for template-matching features, allowing attentional selection to be biased toward external input containing these features (e.g., Desimone \& Duncan, 1995). Given the typicality effects found in our study and in other tasks such as visual search (Maxfield et al., 2014), it is clear that attention is biased toward typical features or exemplars as long as a categorical attention set is maintained. However, what is still unclear is the nature of the target representation. It is possible that categorical attentional sets at least partly consist of visual information, where attention is captured by specific features common to typical exemplars. However, an alternative explanation is that these sets consist of less sensory-dependent conceptual or semantic information, which is more closely linked to typical exemplars. Further research is required to determine which of these explanations better explain why attention is captured by object categories. Future research should also attempt to replicate these findings using eye-tracking to determine whether spatial attention is shifted toward the distractors, as well as whether such shifts vary by typicality.

Overall, our results show that contingent capture can occur at a categorical level, and can interact with an object's typicality. Our results provide a more fine-grained examination of categorical attentional sets, suggesting that attention is particularly biased toward typical exemplars of a category. This extends previous work examining contingent capture for relatively simple features such as color, abrupt onsets, and apparent motion (Folk et al., 1994). Based on these effects, we can conclude that attention is involuntarily shifted towards typical exemplars that belong to the same category as observers' attentional set, even when these items are taskirrelevant.

Acknowledgements This research was supported by a research grant from the Natural Science and Engineering Research Council of Canada Discovery Grant 2016-06359 to Jay Pratt. The authors would like to thank Brett Cochrane for his help with the programming of Experiment 1.

\section{Compliance with ethical standards}

Declaration of interest statement All authors declare they have no conflicts of interest. 


\section{Appendix 1}

Raincloud plots for target-present (a) and target-absent (b) accuracy data in Experiment 1

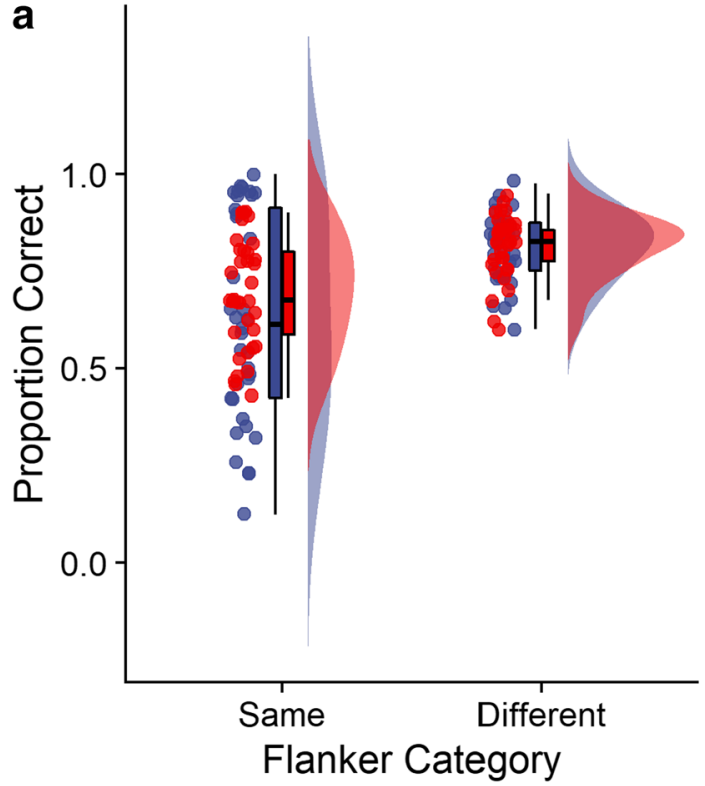

Each plot displays each participant's mean accuracy score per condition, the overall median, the first and third quartiles, and the probability density of the distribution.

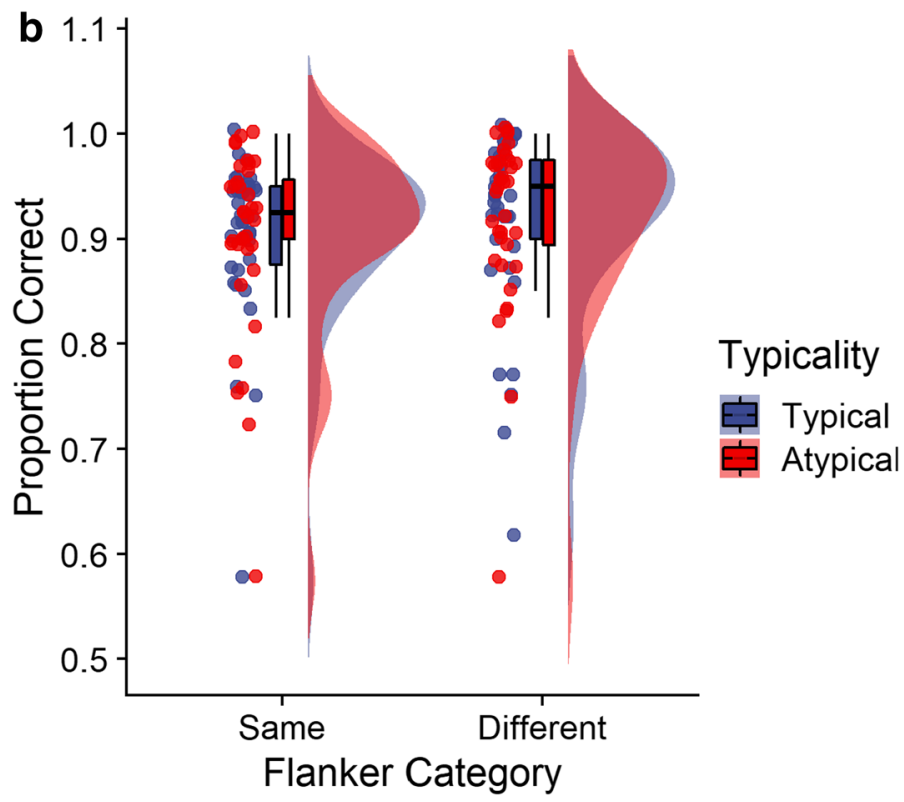

Each plot displays each participant's mean accuracy score per condition, the overall median, the first and third quartiles, and the probability density of the distribution.

\section{Appendix 2}

Raincloud plots for target-present (a) and target-absent (b) accuracy data in Experiment 2
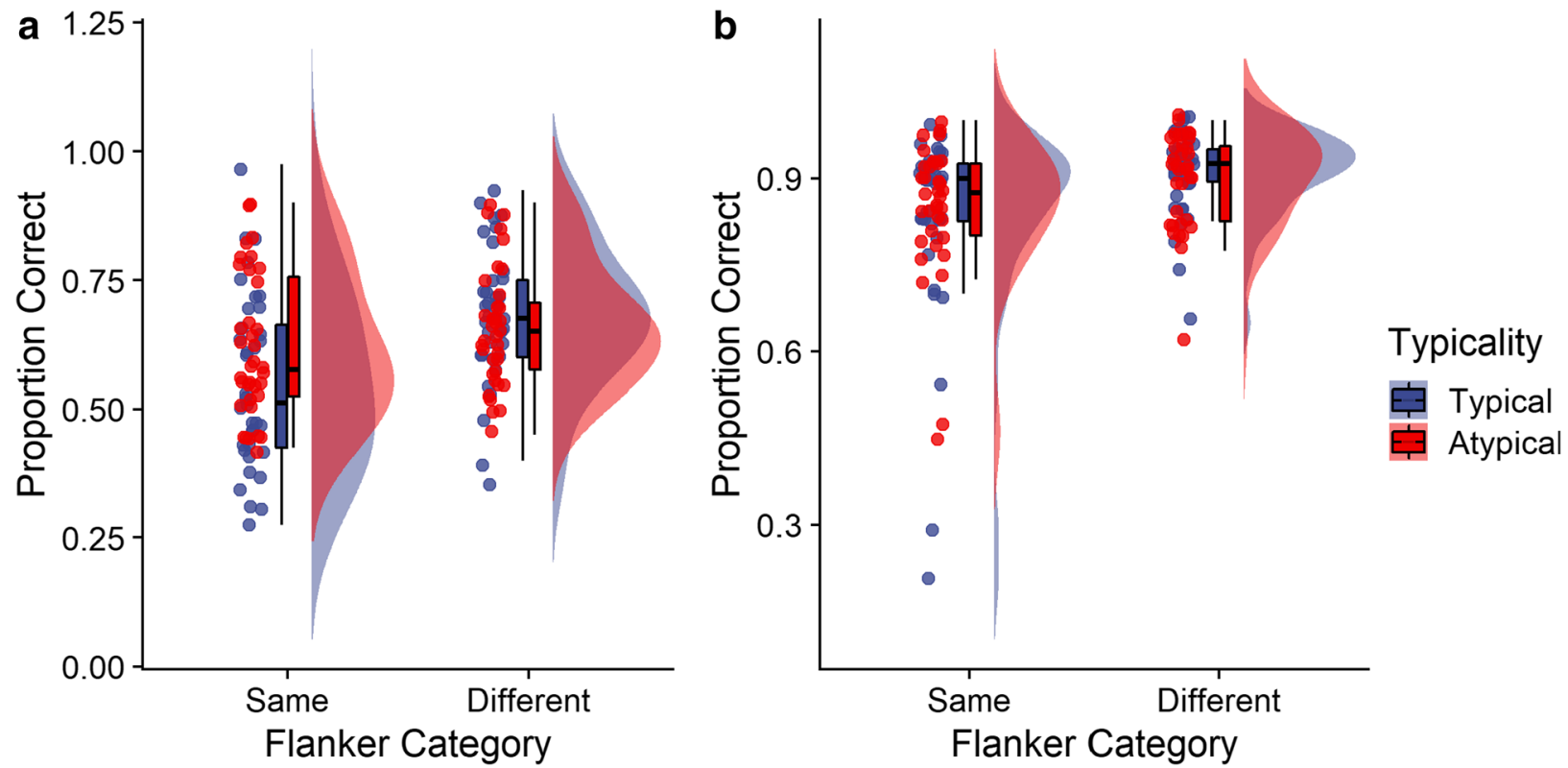


\section{References}

Baier, D., \& Ansorge, U. (2019). Contingent capture during search for alphanumeric characters: A case of feature-based capture or of conceptual category membership? Vision Research, 160, 43-51. https:// doi.org/10.1016/j.visres.2019.02.016

Castelhano, M. S., Pollatsek, A., \& Cave, K. R. (2008). Typicality aids search for an unspecified target, but only in identification and not in attentional guidance. Psychonomic Bulletin \& Review, 15(4), 795801. https://doi.org/10.3758/PBR.15.4.795

Collins, A. M., \& Loftus, E. F. (1975). A spreading-activation theory of semantic processing. Psychological Review, 82(6), 407-428. https:// doi.org/10.1037/0033-295X.82.6.407

Desimone, R., \& Duncan, J. (1995). Neural mechanisms of selective visual attention. Annual Review of Neuroscience, 18, 193-222. https://doi.org/10.1146/annurev.ne.18.030195.001205

Duncan, J., \& Humphreys, G. W. (1989). Visual search and stimulus similarity. Psychological Review, 96(3), 433-458. https://doi.org/ 10.1037/0033-295X.96.3.433

Faul, F., Erdfelder, E., Lang, A.-G., \& Buchner, A. (2007). G*Power 3: A flexible statistical power analysis program for the social, behavioral, and biomedical sciences. Behavioral Research Methods, 39(2), 175-191. https://doi.org/10.3758/BF03193146

Folk, C. L., Leber, A. B., \& Egeth, H. E. (2002). Made you blink! Contingent attentional capture produces a spatial blink. Perception \& Psychophysics, 64(5), 741-753. https://doi.org/10.3758/ BF03194741

Folk, C. L., \& Remington, R. W. (1998). Selectivity in distraction by irrelevant featural singletons: Evidence for two forms of attentional capture. Journal of Experimental Psychology: Human Perception and Performance, 24(3), 847-858. https://doi.org/10.1037/00961523.24.3.847

Folk, C. L., Remington, R. W., \& Johnston, J. C. (1992). Involuntary covert orienting is contingent on attentional control settings. Journal of Experimental Psychology: Human Perception and Performance, 18(4), 1030-1044. https://doi.org/10.1037//0096-1523.18.4.1030

Folk, C. L., Remington, R. W., \& Wright, J. H. (1994). The structure of attentional control: Contingent attentional capture by apparent motion, abrupt onset, and color. Journal of Experimental Psychology: Human Perception and Performance, 20(2), 317-329. https://doi. org/10.1037/0096-1523.20.2.317

Hout, M., Goldinger, S., \& Brady, K. (2014). MM-MDS: A multidimensional scaling database with similarity ratings for 240 object categories from the Massive Memory Picture Database. PLOS ONE, 9(11), 1-11. https://doi.org/10.1371/journal.pone.0112644

Maxfield, J. T., Stalder, W. D., \& Zelinsky, G. J. (2014). Effects of target typicality on categorical search. Journal of Vision, 14(12), 1-11. https://doi.org/10.1167/14.12.1

Maxfield, J. T., \& Zelinsky, G. J. (2012). Searching through the hierarchy: How level of target categorization affects visual search. Visual Cognition, 20(10), 1153-1163. https://doi.org/10.1080/13506285. 2012.735718

Moores, E., Laiti, L., \& Chelazzi, L. (2003). Associative knowledge controls deployment of visual selective attention. Nature Neuroscience, 6(2), 182-189. https://doi.org/10.1038/nn996
Nako, R., Wu, R., Smith, T. J., \& Eimer, M. (2014). Item and categorybased attentional control during search for real-world objects: Can you find the pants among the pans? Journal of Experimental Psychology: Human Perception and Performance, 40(4), 12831288. https://doi.org/10.1037/a0036885

Peirce, J. W., Gray, J. R., Simpson, S., MacAskill, M. R., Höchenberger, R., Sogo, H., Kastman, E., \& Lindeløv, J. (2019). PsychoPy2: Experiments in behavior made easy. Behavior Research Methods, 51, 195-203. https://doi.org/10.3758/s13428-018-01193-y

Rosch, E. (1975). Cognitive representations of semantic categories. Journal of Experimental Psychology: General, 104(3), 192-233. https://doi.org/10.1037/0096-3445.104.3.192

Rosch, E., Simpson, C., \& Miller, R. S. (1976). Structural bases of typicality effects. Journal of Experimental Psychology: Human Perception and Performance, 2(4), 491-502. https://doi.org/10. 1037/0096-1523.2.4.491

Schmidt, J., \& Zelinsky, G. J. (2009). Search guidance is proportional to the categorical specificity of a target cue. Quarterly Journal of Experimental Psychology, 62(10), 1904-1914. https://doi.org/10. 1080/17470210902853530

Theeuwes, J. (1992). Perceptual selectivity for color and form. Perception \& Psychophysics, 51(6), 599-606. https://doi.org/10.3758/ BF03211656

Willenbockel, V., Sadr, J., Fiset, D., Horne, G. O., Gosselin, F., \& Tanaka, J. W. (2010). Controlling low-level image properties: The SHINE Toolbox. Behavior Research Methods, 42(3), 671-684. https://doi.org/10.3758/BRM.42.3.671

Wolfe, J. M. (1994). Guided Search 2.0: A revised model of visual search. Psychonomic Bulletin \& Review, 1(2), 202-238. https://doi.org/10. 3758/BF03200774

Wu, R., Scerif, G., Aslin, R. N., Smith, T. J., Nako, R., \& Eimer, M. (2013). Searching for something familiar or novel: ERP correlates of top-down attentional selection for specific items or categories. Journal of Cognitive Neuroscience, 25(5), 719-729. https://doi. org/10.1162/jocn_a_00352

Wyble, B., Folk, C., \& Potter, M. C. (2013). Contingent attentional capture by conceptually relevant images. Journal of Experimental Psychology: Human Perception and Performance, 39(3) 861-871. https://doi.org/10.1037/a0030517

Yang, H., \& Zelinsky, G. J. (2009). Visual search is guided to categorically-defined targets. Vision Research, 49(16), 20952103. https://doi.org/10.1016/j.visres.2009.05.017

Yantis, S., \& Jonides, J. (1984). Abrupt visual onsets and selective attention: Evidence from visual search. Journal of Experimental Psychology: Human Perception and Performance, 10(5), 601621. https://doi.org/10.1037//0096-1523.10.5.601

Open practices statement The data and materials for this research are available at https://osf.io/xjk4f/. This research was not preregistered.

Publisher's note Springer Nature remains neutral with regard to jurisdictional claims in published maps and institutional affiliations. 